\author{
Marquette University \\ e-Publications@Marquette
}

School of Dentistry Faculty Research and

Publications

Dentistry, School of

$1-4-2011$

\title{
Apoptosis in Displaced Temporomandibular Joint Disc with and without Reduction: An Immunohistochemical Study
}

\author{
Carla Loreto \\ University of Catania \\ Luis Eduardo Almeida \\ Marquette University, luis.almeida@marquette.edu \\ Paula C. Trevilatto \\ University of Panarà \\ Rosalia Leonardi \\ University of Catania
}

Follow this and additional works at: https://epublications.marquette.edu/dentistry_fac

Part of the Dentistry Commons

\section{Recommended Citation}

Loreto, Carla; Almeida, Luis Eduardo; Trevilatto, Paula C.; and Leonardi, Rosalia, "Apoptosis in Displaced Temporomandibular Joint Disc with and without Reduction: An Immunohistochemical Study" (2011). School of Dentistry Faculty Research and Publications. 338.

https://epublications.marquette.edu/dentistry_fac/338 


\section{Marquette University}

\section{e-Publications@Marquette}

\section{Dentistry Faculty Research and Publications/School of Dentistry}

This paper is NOT THE PUBLISHED VERSION; but the author's final, peer-reviewed manuscript. The published version may be accessed by following the link in the citation below.

Journal of Oral Pathology \& Medicine, Vol. 40, No. 1 (January 2011): 103-110. DOI. This article is (C) Wiley and permission has been granted for this version to appear in e-Publications@Marquette. Wiley does not grant permission for this article to be further copied/distributed or hosted elsewhere without the express permission from Wiley.

\section{Apoptosis in displaced temporomandibular joint disc with and without reduction: an immunohistochemical study}

Carla Loreto

Department of Anatomy, Diagnostic Pathology, Forensic Medicine, Hygiene and Public Health, University of Catania, Catania, Italy

Luis Eduardo Almeida

School of Dentistry, Marquette University, Milwaukee, WI, USA

Center for Health and Biological Sciences, Pontifical Catholic, University of Panarà, Curitibe, Brazil

Paula Trevilatto

Center for Health and Biological Sciences, Pontifical Catholic, University of Panarà, Curitibe, Brazil

Rosalia Leonardi

Department of Dentistry, Faculty of Dentistry, University of Catania, Policlinico Universitario, Catania, Italy

\section{Abstract}

Internal derangement (ID) of the temporomandibular joint (TMJ) is due to an abnormal relationship of the articular disc to the mandibular condyle, glenoid fossa and articular eminence. The two most common types of internal derangement are anterior disc displacement with (ADDwR) and without reduction (ADDwoR). Disc 
displacement is associated with degenerative tissue changes. The histological features of discs from patients with TMJ ID reflect a general remodelling caused by abnormal loading. A correlation has been demonstrated between TMJ ID and apoptosis. Few investigations have addressed the role of apoptosis or caspase activity in TMJ ID. The apoptosis activation process was studied in different areas of discs from 18 patients with ID (both ADDwR and ADDwoR) and four cadavers (controls), with emphasis on the expression of caspase 3, whose activation makes the death process irreversible. The results showed a greater proportion of caspase 3-positive cells in ADDwR and ADDwoR than in control discs. Immunopositivity also varied between disc areas; in particular, in ADDwoR sections labelled cells were significantly more numerous $(P<0.01)$ in the posterior disc attachment than in the anterior and intermediate bands. In addition, a significantly greater proportion of labelled cells was seen in the anterior $(+)$ and intermediate $(++)$ band of ADDwR compared with ADDwoR discs both bands $(P<0.05)$. These data suggest the importance of programmed cell death in the progression of TMJ ID.

\section{Keywords}

caspase 3, internal derangement, temporomandibular joint disc

\section{Introduction}

Internal derangement (ID) of the temporomandibular joint (TMJ) is due to an abnormal relationship of the articular disc to the mandibular condyle, glenoid fossa and articular eminence. The two most common types of internal derangement are anterior disc displacement with (ADDwR) and without reduction (ADDwoR). In ADDwR the disc slides into and out of its normal functional position as the jaw opens and closes, with an early click due to the residual elasticity of the bilaminar zone and collateral ligaments; in ADDwoR the disc glides anteriorly to a lower rest position, remaining stuck in the anterior joint recess and failing to revert to its normal position with condylar movement $(\underline{1}, 2)$. If the disc slips out of place or is displaced, it can prevent proper condyle movement and cause dysfunction. The disc can deform, becoming misshapen or even torn ( $\underline{3})$.

Internal derangement (ID) manifests clinically as pain, joint sounds, muscle tenderness and limitation of mouth opening (4). Disc displacement seems to have a large role in causing signs, symptoms and osteoarthritic changes in TMJ (므). Although TMJ ID affects $20-25 \%$ of the population $(\underline{6}, \underline{7})$, there are few data on the biochemical components of the displaced disc. The histological features of discs removed from patients with TMJ ID ( $\underline{8}-\underline{12})$ reflect a general remodelling caused by abnormal loading (ㅇ). Degenerative joint changes are known to be influenced by the type and degree of disc displacement and to start in the posterior disc attachment (PDA) (1316); advanced ID corresponds to a deteriorated disc configuration (17).

It has been speculated that excessive mechanical stress damages TMJ disc tissue directly or indirectly $(\underline{18}, \underline{19})$.

Disc displacement is associated with degenerative tissue changes $(\underline{8}, \underline{9}, \underline{11}, \underline{20}-\underline{26})$, but the underlying mechanisms remain unclear. Although previous research has demonstrated a correlation between TMJ ID and apoptosis (27-30), most studies have been done in animal models because of the difficulty in obtaining human material. Chondrocyte apoptosis is increased in articular cartilage from displaced rabbit disc, above all in the proliferative and hypertrophic zones ( $\underline{31})$, suggesting that apoptotic TMJ changes are closely related to inflammation and overload conditions. In knee and ankle cartilage from patients with osteoarthritis (OA) and rheumatoid arthritis, apoptotic chondrocytes increase significantly with disease aggravation $(\underline{32}, \underline{33})$. There are few investigations of the role of apoptosis in $\operatorname{TMJ} I D(\underline{15}, \underline{27}, \underline{29})$ and none providing data on caspase activity in TMJ ID discs from both ADDWR and ADDwoR patients.

Apoptosis is physiologically involved in various aspects of mammalian development, including embryogenesis, normal tissue turnover and homeostasis. This fine process is activated by two main mechanisms, the intrinsic, mitochondria-mediated pathway and the extrinsic pathway, induced by death signaling ligands, e.g. TNFa and Fas Ligand (FasL), which bind to their receptors (4-37). The ultimate executioner of apoptotic cell death is a 
group of proteins called caspases $(\underline{38}, \underline{39})$. Caspases (cysteine-containing aspartate-specific proteases) are enzymes that cleave proteins, resulting in cell destruction. There are several caspases acting either as inducer/initiators (caspase 2, 8, and 9) or as executioners (caspase 3, 6, and 7) (40). In particular, once caspase 3 is activated, the death process becomes irreversible.

In this study we tested the hypothesis that the apoptosis mechanism has an important role in the progression of TMJ ID. The apoptosis activation process was therefore studied in different areas of discs from patients with ID (both ADDwR and ADDwoR), with emphasis on caspase 3 expression.

\section{Materials and methods}

\section{Patients and tissues}

Surgical TMJ disc specimens from 14 female and four male patients aged 24-41 years (mean $34.2 \quad \pm \quad 5.4$ ) were obtained from the archives of the University of the Pontifical Catholic University of Paraná, Brazil. ID was confirmed by clinical history, physical examination and magnetic resonance imaging (MRI). The study was approved by the local ethics committee. The informed consent of each patient was obtained before tissue collection. Patients' sex, age distribution and symptom severity are detailed in Tables 1 and 2. Unassisted maximum mouth opening ( $\mathrm{MMO}$ ) was measured with a millimetre ruler as the interincisal distance on maximum mouth opening as a measure of disease severity. Pain intensity in the preceding week was measured on a 100 visual analogue scale (VAS) with anchor points 0 for 'no pain' and 100 for the 'worst imaginable pain'.

Table 1. Clinical characteristics of the study group with TMJ internal derangement

\begin{tabular}{|c|c|c|c|c|c|c|}
\hline Patient & Ethnicity & Gender & Age (years) & Diagnosis & Affected side & \\
\hline & & & & & Right & Left \\
\hline 1 & Caucasian & Female & 33 & ADDwR & $x$ & \\
\hline 2 & Caucasian & Female & 38 & ADDwR & $x$ & \\
\hline 3 & Native American & Female & 29 & ADDwR & & $\mathrm{x}$ \\
\hline 4 & African Brazilian & Female & 36 & ADDwR & $X$ & \\
\hline 5 & Native American & Female & 24 & ADDwR & & $\mathrm{x}$ \\
\hline 6 & Caucasian & Female & 41 & ADDwR & & $x$ \\
\hline 7 & Native American & Male & 37 & ADDwR & & $x$ \\
\hline 8 & Caucasian & Female & 28 & ADDwR & $\mathrm{x}$ & \\
\hline 9 & Caucasian & Female & 25 & ADDwR & $x$ & \\
\hline 10 & Native American & Female & 34 & ADDwR & & $x$ \\
\hline 11 & Native American & Female & 35 & ADDwR & $\mathrm{x}$ & \\
\hline 12 & Caucasian & Female & 40 & ADDwR & $x$ & \\
\hline 13 & African Brazilian & Male & 33 & ADDwR & $x$ & \\
\hline 14 & African Brazilian & Female & 36 & ADDwoR & & $\mathrm{x}$ \\
\hline 15 & Native American & Female & 28 & ADDwoR & & $x$ \\
\hline 16 & Caucasian & Male & 41 & ADDwoR & $x$ & \\
\hline 17 & Caucasian & Female & 40 & ADDwoR & $x$ & \\
\hline 18 & African Brazilian & Female & 39 & ADDwoR & & $x$ \\
\hline
\end{tabular}

$A D D w R$, anterior disc displacement with reduction; ADDwoR, anterior disc displacement without reduction.

Table 2. Visual analogue scale (VAS); anchor points 0 ('no pain') and 100 ('worst imaginable pain')

\begin{tabular}{|l|c|c|c|c|l|l|}
\hline & Age & & MMO & VAS & \\
\hline & MV & SD & MV & SD & MV & SD \\
\hline ADDwR & 32.7 & 5.5 & 35.6 & 5.1 & 78 & 6 \\
\hline ADDwoR & 36.5 & 4.4 & 27.3 & 4.3 & 87 & 7 \\
\hline
\end{tabular}


The diagnosis that led to disc excision was painful disc derangement with impaired function.

Discectomy has come under increasing attack as the standard therapy for disc displacement, as a number of surgeons now favour a conservative approach; however, other researchers still believe in the value of discectomy (41-43). Open surgery for patients with ID therefore has an ethical justification. The authors used this small sample of whole TMJ discs because they provide exhaustive information on their degree of degeneration.

Inclusion criteria were unsuccessful non-surgical treatment; a diagnosis of TMJ ID; and tenderness to TMJ palpation or interference with mandibular movements. Exclusion criteria were other TMJ disorders, dentofacial deformity; major jaw trauma; previous TMJ surgery; and previous steroid injections in the TMJ.

Discs were divided into those with (ADDwR) and without disc reduction (ADDwoR). After surgical excision, all discs were found to be well conserved; they were macroscopically deformed and none had a normal biconcave shape. The anterior and intermediate band and the PDA were preserved in all specimens.

Four virtually unaffected human TMJ discs from cadavers of the collection of the Department of Anatomy of Catania University, Catania, Italy, were studied along with the diseased discs. These paraffin-embedded autopsy specimens, obtained from one male and three female subjects (mean age: $49.7 \quad \pm \quad 4.4$ years), were selected because the donors' clinical histories were negative for generalized joint disease or TMJ arthropathy; none had macroscopic signs of degenerative or inflammatory joint disease on dissection and none were displaced. They stained with Harry's haematoxylin on light microscopy and exhibited no detectable pathological changes.

\section{Immunohistochemistry}

The 18 surgical specimens were fixed overnight in 10\% neutral buffered formalin (Bio-Optica, Milan, Italy). After fixation and overnight washing they were sectioned through the centre along the parasagittal plane, perpendicular to the long axis. Each tissue block was dehydrated in graded ethanol and embedded in paraffin, preserving anatomical orientation. Specimens were cut into $5 \mu \mathrm{m}$ thick sections and placed on silanized glass slides for routine haematoxylin staining and immunohistochemistry. Sections were irradiated $(5 \min \times 3)$ in capped polypropylene slide-holders with citrate buffer $(\mathrm{pH} \mathrm{6})$, using a microwave oven $(750 \mathrm{~W})$ to unmask antigenic sites.

A rabbit polyclonal anti-caspase 3 antibody (Cell Signaling Technology, Inc., Denvers, MA, USA) used at 1:200 working dilution was applied on each section and incubated overnight at $+4^{\circ} \mathrm{C}$ in a moist chamber. Immunocomplexes were detected by incubation with the secondary antibody and then with streptavidinperoxidase complexes for 15 min each at room temperature (LSAB2/HRP kit; DAKO, Glostrup, Denmark). After rinsing in three changes of PBS, the immunoreaction was visualized by incubating sections in a $0.1 \% 3,3^{\prime}$ diaminobenzidine and $0.02 \%$ hydrogen peroxide solution (DAB substrate kit, Vector Laboratories, Burlingame, CA, USA) for 4 min. Sections were lightly counterstained with Meyer's haematoxylin.

\section{Immunohistochemical evaluation}

Immunostained slides were evaluated on a consensus basis by two anatomists, who were blinded to patient identity, clinical status and group identification, using a double-headed light microscope.

Staining intensity and the proportion of caspase 3-immunopositive cells were assessed and recorded. Three different TMJ disc areas were analysed: the anterior band, the intermediate zone, and the PDA. Staining intensity (SI) was graded on a 0-4 scale: 0: no detectable staining; 1 : weak staining; 2 : moderate staining; 3 : 
strong staining; 4: very strong staining. Caspase 3-positive cells, observed at $200 \times$ magnification, were scored as a proportion of 200 cells as 0: $<5 \%$; 1: 6-30\%; 2: 31-50\%; 3: >50\%; and 4: $>75 \%$ (extent score, ES).

The final staining score (FSS) was the sum of SI and ES.

\section{Positive and negative controls}

This was performed to test the specific reaction of the primary antibody at the protein level. For positive control tests, hepatocellular carcinoma tissue was exposed to immunoperoxidase. The strong caspase 3

immunolabelling had a cytoplasmic localization. For negative controls, randomly selected TMJ disc sections were treated with normal rabbit serum instead of the specific antibody.

\section{Statistical analysis}

All experiments were performed at least in triplicate.

Data were analysed using the Mann-Whitney $U$-tests; $P<0.05$ were considered significant. The MannWhitney $U$-test was also used to compare protein expression among disc specimens. Comparisons were carried out for the anterior and intermediate bands and the PDA in ADDwR and ADDwoR patients. All data were analysed with the spss program (SPSS ${ }^{\circledR}$ release 16.0 , Chicago, IL, USA).

\section{Results}

All patients had previously undergone unsuccessful conservative treatment including bite splints, physical therapy, chiropractic manipulation, drug therapy and vitamin supplements, for at least 6 months.

Haematoxylin staining showed abnormal collagen fibre arrangement and collagen bundle fragmentation and tearing in diseased TMJ discs and preservation of multidirectional collagen bundles in control discs. The damaged discs also showed diminished cellularity and an altered cell population ratio with an increase in chondrocyte-like cells related to the severity of the morphological disc damage, and decreased fibroblast-like cells that was noted especially in ADDwR discs.

Immunohistochemical caspase 3 labelling was demonstrated in all disc sections, with different patterns of reactivity in the different areas and patient groups. The immunoreactivity was detected in the cell cytoplasm; in particular, fibroblast- and chondrocytes-like cells were strongly positive (SI score $=3$ ).

A greater proportion of caspase 3-positive cells was demonstrated in ADDWR and ADDwoR than in normal discs (Table 3). Immunopositivity also varied between disc areas; in particular, in ADDwoR sections labelled cells were significantly more numerous $(P<0.01)$ in the PDA (Figs 1 and 4$)$ than in the anterior (Figs 2 and 5 ) and intermediate bands (Figs 3 and 6 ). In addition, a significantly greater proportion of labelled cells was seen in the anterior (+) and intermediate (++) band of ADDwR compared with ADDwoR discs both bands $(P<0.05)$.

Table 3. Final caspase 3 immunolabelling staining score (sum of staining intensity and extent scores)

\begin{tabular}{|c|c|c|c|c|c|c|c|c|c|c|}
\hline Patient & Diagnosis & $\begin{array}{c}\text { Anterior } \\
\text { band }\end{array}$ & & & $\begin{array}{l}\text { Intermediate } \\
\text { band }\end{array}$ & & & $\begin{array}{l}\text { Posterior disc } \\
\text { attachment }\end{array}$ & & \\
\hline & & FSS & SI & ES & FSS & SI & ES & FSS & SI & ES \\
\hline 1 & ADDwR & 4 & 2 & 2 & 4 & 2 & 2 & 6 & 3 & 3 \\
\hline 2 & ADDwR & 4 & 2 & 2 & 3 & 2 & 1 & 7 & 4 & 3 \\
\hline 3 & ADDwR & 2 & 1 & 1 & 3 & 2 & 1 & 6 & 3 & 3 \\
\hline 4 & ADDwR & 3 & 2 & 1 & 5 & 2 & 3 & 5 & 2 & 3 \\
\hline 5 & ADDwR & 3 & 2 & 1 & 4 & 1 & 3 & 6 & 3 & 3 \\
\hline
\end{tabular}




\begin{tabular}{|l|l|l|l|l|l|l|l|l|l|l|}
\hline 6 & ADDwR & 3 & 1 & 2 & 3 & 2 & 1 & 5 & 2 & 3 \\
\hline 7 & ADDwR & 4 & 2 & 2 & 4 & 2 & 2 & 5 & 3 & 2 \\
\hline 8 & ADDwR & 4 & 1 & 3 & 4 & 2 & 2 & 7 & 3 & 4 \\
\hline 9 & ADDwR & 2 & 1 & 1 & 6 & 2 & 4 & 7 & 3 & 4 \\
\hline 10 & ADDwR & 3 & 2 & 1 & 5 & 2 & 3 & 6 & 3 & 3 \\
\hline 11 & ADDwR & 4 & 3 & 1 & 5 & 2 & 3 & 6 & 3 & 3 \\
\hline 12 & ADDwoR & 3 & 2 & 1 & 4 & 2 & 2 & 8 & 4 & 4 \\
\hline 13 & ADDwoR & 4 & 2 & 2 & 4 & 2 & 2 & 7 & 4 & 3 \\
\hline 14 & ADDwoR & 3 & 2 & 1 & 6 & 4 & 2 & 7 & 2 & 4 \\
\hline 15 & ADDwoR & 5 & 2 & 3 & 5 & 2 & 3 & 7 & 3 & 4 \\
\hline 16 & ADDwoR & 3 & 1 & 2 & 6 & 3 & 3 & 7 & 3 & 4 \\
\hline 17 & ADDwoR & 5 & 2 & 3 & 6 & 2 & 4 & 7 & 2 & 5 \\
\hline 18 & ADDwoR & 4 & 2 & 2 & 4 & 3 & 1 & 6 & 3 & 3 \\
\hline 19 & Control & 2 & 1 & 1 & 3 & 2 & 1 & 2 & 1 & 1 \\
\hline 20 & Control & 1 & 1 & 0 & 2 & 1 & 1 & 3 & 2 & 1 \\
\hline 21 & Control & 2 & 1 & 1 & 2 & 1 & 1 & 2 & 1 & 1 \\
\hline 22 & Control & 2 & 1 & 1 & 2 & 2 & 0 & 2 & 1 & 1 \\
\hline
\end{tabular}

Staining intensity (0: no detectable staining, 1: weak staining, 2: moderate staining, 3: strong staining, 4: very strong staining) and proportion of labelled cells (extent score: 0: $<5 \% ; 1: 6-30 \% ; 2: 31-50 \% ; 3:>50 \% ; 4:>75 \%$ ). ADDwR, anterior disc displacement with reduction; ADDwoR, anterior disc displacement without reduction.

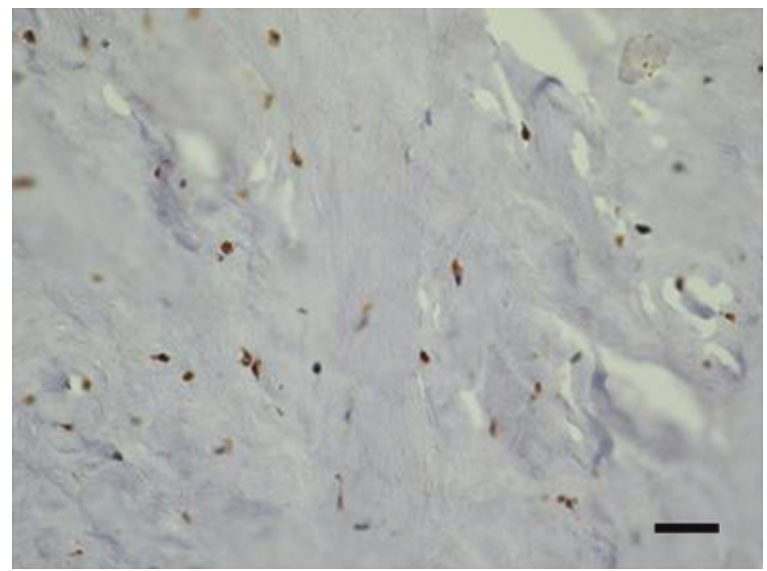

Figure 1

Posterior attachment of ADDwoR disc. Almost all cells are caspase 3-immunopositive. Bar: $100 \mu$ m (40x). 


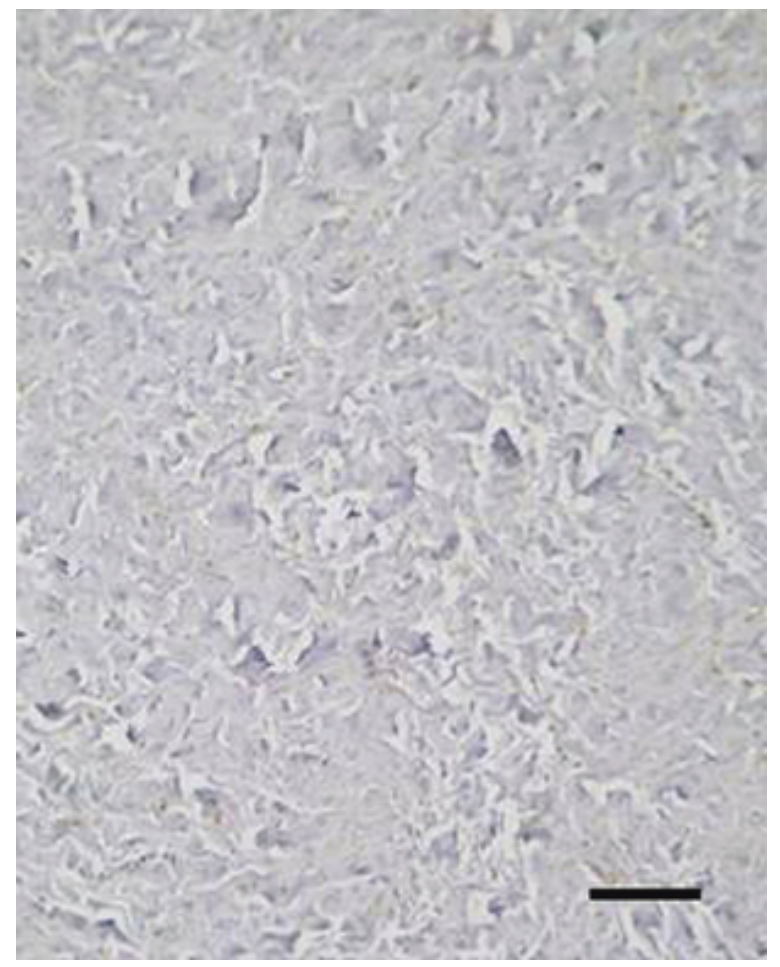

\section{Figure 4}

Posterior attachment of ADDwoR disc. Bar: $100 \mu \mathrm{m}(10 \times)$.

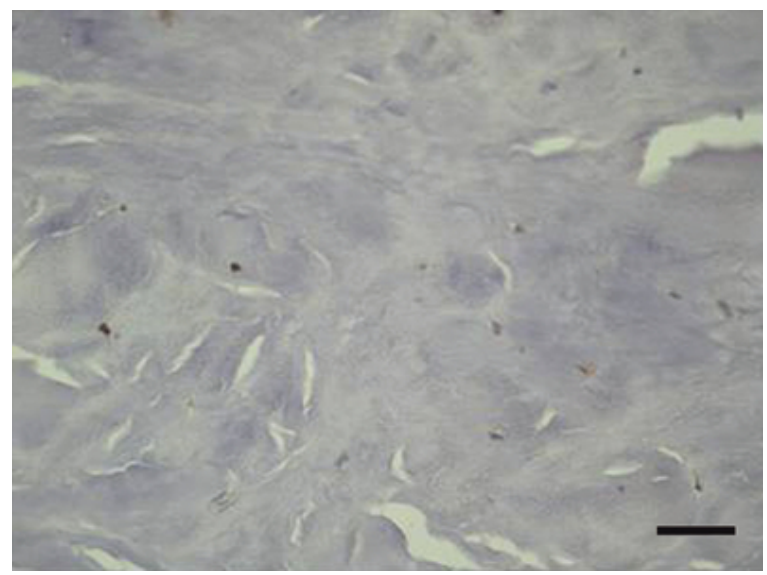

\section{Figure 2}

Caspase 3 positivity in anterior band of ADDwoR disc. Bar: $100 \mu \mathrm{m}(40 \times)$. 


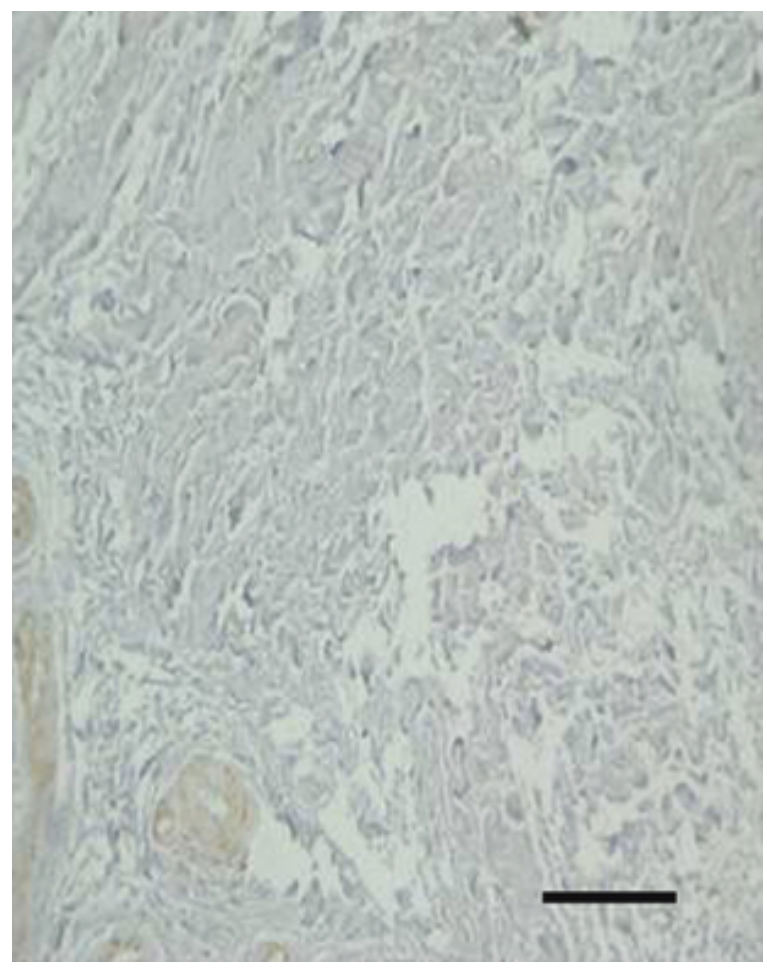

\section{Figure 5}

Caspase 3 positivity in anterior band of ADDwoR disc. Bar: $100 \mu \mathrm{m}(10 \times)$.

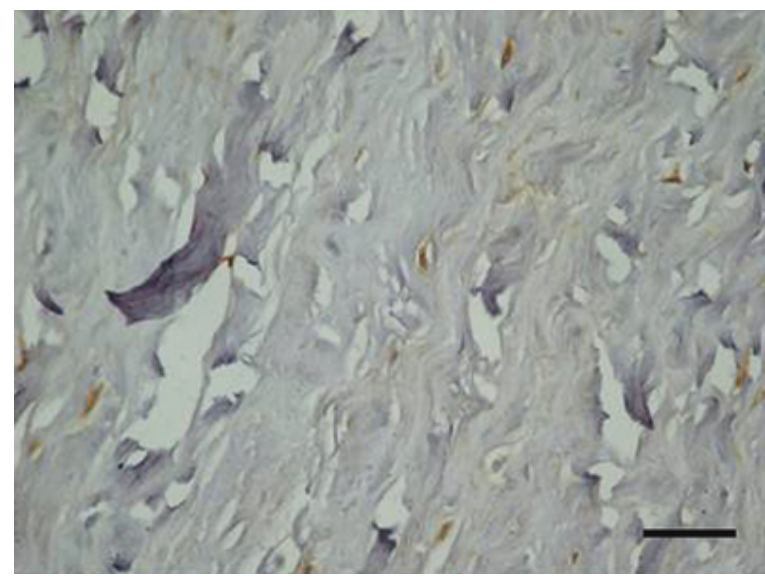

\section{Figure 3}

Intermediate band of ADDwoR disc staining for anti-caspase 3 antibody. Bar: $100 \mu \mathrm{m}(40 \times)$ 


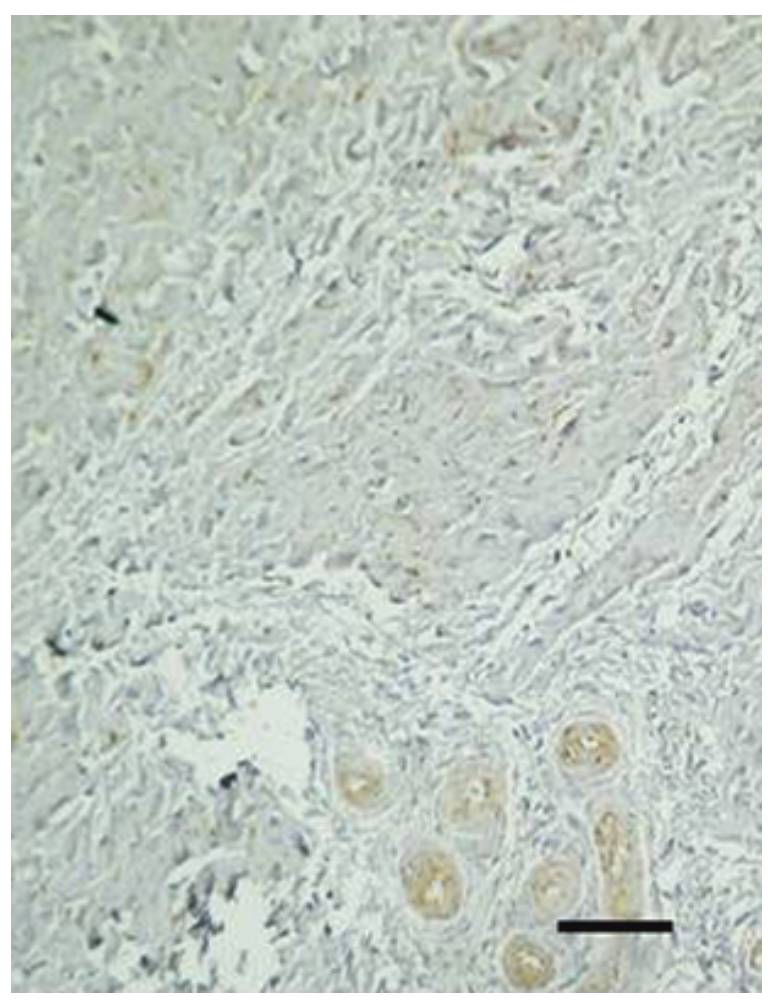

\section{Figure 6}

Intermediate band of ADDwoR disc staining for anti-caspase 3 antibody. Bar: $100 \mu \mathrm{m}(10 \times)$.

No difference between the disc regions was seen in normal specimens.

Control discs exhibited few scattered caspase 3-immunolabelled cells without significant differences among the three areas.

\section{Discussion}

Joints are subject to a variety of functional loads and continuously adapt to changing functional demands to maintain structural and functional integrity. TMJ disc displacement is associated with a general remodelling response caused by abnormal disc loading by the condyle ()ㅡ. Even though the TMJ is not a weight-bearing joint, the forces acting on it are nonetheless considerable (44)).

The different proportions of caspase 3-positive cells found in the three regions of diseased compared with normal discs are explained by the fact that the maximum compressive stresses in pathological discs are exerted on the PDA, due to disc displacement during mouth opening. In addition, immunolabelling patterns also differed between ADDwR and ADDwoR discs. In the former, before disc recapture, the maximum stresses peak in the anterior band ( $\underline{1})$; then, when the disc is reduced they are exerted on intermediate zone, where the proportion of caspase 3-positive cells was in fact highest. In ADDwoR the maximum compressive stresses are exerted on the PDA (1), where we documented a greater caspase 3 expression compared with the other two bands.

These pathophysiological considerations account at least partially for the different proportions of caspase 3positive cells found in the posterior, intermediate and anterior bands.

Disc position and degeneration are closely related; mechanical overloading enhances the degeneration, as cells sense the excessive load and convert it to biological signals that result in specific tissue responses $(\underline{13}, \underline{45})$. It is also well known that degenerative TMJ disorders include both OA with tissue inflammation and non- 
inflammatory osteoarthrosis (46). Damage to the TMJ articular cartilage is associated with catabolic activity due to proinflammatory cytokine activity. Several proinflammatory cytokines, such as TNF $\alpha$, interleukin (IL)-1 $\beta$, IL-6,

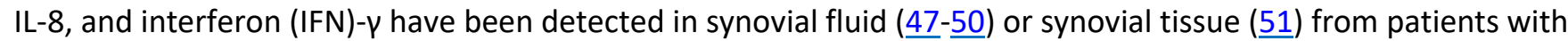
TMJ ID and OA. In particular, IL-1 $\beta$ and TNF $\alpha$ seem to be involved in TMJ ID development and to play a coordinated role in its pathogenesis (52). The inflammation cascade alters the extracellular matrix (ECM) by depleting matrix substances, such as collagen (53). Recently, nitric oxide, an important regulator of inflammation $(\underline{54}, \underline{55})$, has also been proved to be a modulator of apoptosis $(\underline{56}, \underline{57})$. In particular, apoptosis caused by oxidative stress is known to be involved in inflammatory joint diseases $(\underline{58}, \underline{59})$. Tightly controlled homeostatic mechanisms are required to regulate the balance between proliferative activity (increase in cell number) and apoptotic activity (decrease in cell number) for the functional and structural integrity of organs and tissue to be maintained ( $\underline{60})$. Apoptosis can be activated by a diverse group of signals including oxidative damage, presence (e.g. TGF $\beta$ ) or absence (e.g. CSF) of specific growth factors, ligand binding to receptors (e.g. TNF $\alpha$ ), disruption of cell-cell or cell-matrix interactions (61). In particular TNF $\alpha$ has been demonstrated to modulate apoptosis in various tissues and chondrocytes in rabbit TMJ (62). Damaged discs show histomorphological changes that result in altered cell population ratios, abnormal collagen fiber arrangement, fragmentation of collagen fibrils, meniscal tears with new vessel formation, mucoid degeneration and disc hyalinization. The more advanced the ID, the more deteriorated the disc configuration (17). It has also been demonstrated that in patients with ADDwoR increased apoptosis of the synovium might contribute to TMJ degeneration (흐).

Previous studies of degenerated intervertebral disc (IVD) have demonstrated that insufficient restoration of functional ECM equilibrium is strongly associated with cell apoptosis (느). Apoptosis may be a major factor in cell number reduction in disc aging and degeneration (65). An excess of programmed death of disc cells is also a potential cause of degenerative disc disease $(\underline{65}, \underline{66})$. In synthesis, if apoptotic cells are not removed they may lead to inflammatory responses that can promote chronic inflammatory conditions, as shown in an animal model of acute TMJ inflammation $(\underline{29}, \underline{67})$. Immunohistological findings of apoptosis-related factors suggest that the apoptotic reaction is associated with the progression of TMJ disease (2 28$)$.

Few studies have investigated the possible role of apoptosis in TMJ disease and its relation to the degree of disc displacement.

Evidence of a dose-dependent relationship between apoptosis and mechanical overload has also been provided in herniated IVD ( $\underline{68})$. The highest amount of cell death in IVD has been observed in the areas most affected by disc deformation $(\underline{13}, \underline{14}, \underline{40})$. Moreover, increased caspase 3 gene transcription has been demonstrated in vertebral endplate trauma (69).

Caspase 3 is commonly activated by caspase 8 via a receptor-mediated extrinsic pathway, but can also be activated via an intrinsic pathway involving caspase 9 and other factors $(\underline{29}, \underline{64})$. We previously described the immunohistochemical overexpression of tumour necrosis factor-related apoptosis inducing ligand (TRAIL) and its death receptor DR5 in the same 18 discs, documenting the activation of the extrinsic pathway (15). In addition, analysis of the presence and regional distribution of these two molecules in discs with and without reduction documented a significantly greater proportion of immunostained cells in both types of specimens compared with normal discs, with regional variations that depended on disc portion (greater in the PDA than in the anterior or intermediate bands of both ADDwR and ADDwoR discs) (70). The apoptotic cascade is divided into three sets of stages. The initiation stages include induction of the cascade, for instance by ligand-receptor interactions leading to the first proteolytic event. The execution stages begin with the activation of execution caspases such as caspase 3: this is called the point of no return because, once activated, these proteases degrade a variety of proteins, resulting in irreversible cell damage. These complex events lead to apoptotic death, with collapse of the nucleus and of the cell itself (61). The present study was also conducted to gain insights into apoptotic cell death, with caspase 3 activation as the hallmark of the point of no return. 
A greater proportion of caspase 3-labelled cells was detected in the PDA compared to the anterior and intermediate zones of both ADDwR and ADDwoR discs, demonstrating that disc degeneration starts in this area, as previously shown by Orhan et al. (16). However, our data document a different involvement of the intermediate and anterior bands compared with that work. Indeed in these two bands caspase 3immunolabelled cells were more numerous in ADDwR than in ADDwoR discs, suggesting that the mechanical strain of mandibular movement may be stimulated by the disc's sliding into and out of its normal position as the jaw opens and closes, inducing apoptosis activation in these areas. The phenomenon thus may configure an endogenous reaction aimed to restore the homeostasis and/or remodel the organ. Ex vivo studies with loadchallenged murine IVD chondrocytes and fibrocytes demonstrated a strong correlation of impact amplitude and duration with the degree of apoptosis-induced disc cell degeneration $(\underline{71}, \underline{72})$.

In conclusion, the present study provided information on apoptosis-induced irreversible cell death in displaced TMJ discs with and without reduction, and suggests that it is determinating for the topography of the subsequent disc damage and for the progression of this common disorder.

\section{References}

1. Pérez del Palomar, A, Doblaré, M. An accurate simulation model of anteriorly displaced TMJ discs with and without reduction. Med Eng Phys 2007; 29: 216- 26.

2. Okeson, JP Management of temporomandibular disorders and occlusion. St Louis: Mosby-Year book, Inc, 1993; 294, 409, 477.

3. Eberhard, D, Bantleon, HP, Steger, W. Functional magnetic resonance imaging of temporomandibular joint disorders. Eur J Orthod 2000; 22: 489- 97.

4. Nitzan, DW. The process of lubrication impairment and its involvement in temporomandibular joint disc displacement: a theoretical concept. J Oral Maxillofac Surg 2001; 59: 36- 45.

5. Wilkes, $\mathrm{CH}$. Structural and functional alterations of the temporomandibular joint. Northwest Dent 1978; 57: 287-94.

6. Detamore, MS, Athanasiou, KA. Structure and function of the temporomandibular joint disc: implications for tissue engineering. J Oral Maxillofac Surg 2003; 61: 494- 506.

7. Pankov, R, Yamada, KM. Fibronectin at a glance. J Cell Sci 2002; 115: 3861- 3.

8. Scapino, RP. Histopathology associated with malposition of the human temporomandibular joint disc. Oral Surg Oral Med Oral Pathol 1983; 55: 382- 97.

9. Hall, MB, Brown, RW, Baughman, RA. Histologic appearance of the bilaminar zone in internal derangement of the temporomandibular joint. Oral Surg Oral Med Oral Pathol 1984; 58: 375- 81.

10. Isacsson, G, Isberg, A, Johansson, AS, Larson, O. Internal derangement of the temporomandibular joint: radiographic and histologic changes associated with severe pain. J Oral Maxillofac Surg 1986; 44: 771-8.

11. McCoy, JM, Gotcher, JE, Chase, DC. Histologic grading of TMJ tissues in internal derangement. Cranio 1986; 4: $213-18$.

12. Kurita, K, Westesson, PL, Sternby, NH, et al. Histologic features of the temporomandibular joint disk and posterior disk attachment: comparison of symptom-free persons with normally positioned disks and patients with internal derangement. Oral Surg Oral Med Oral Pathol 1989; 67: 635- 43.

13. Westesson, PL, Bronstein, SL, Liedberg, J. Internal derangement of the temporomandibular joint: morphologic description with correlation to joint function. Oral Surg Oral Med Oral Pathol 1985; 59: 32331.

14. Leonardi, R, Loreto, C, Barbato, E, Polimeri, A, Caltabiano, R, Lo Muzio, L. A histochemical survey of the human temporomandibular joint disc of patients with internal derangement without reduction. J Craniofac Surg 2007; 18: 1429- 33.

15. Loreto, C, Musumeci, G, Leonardi, R. Chondrocyte-like apoptosis in temporomandibular joint disc internal derangement as a repair-limiting mechanism. An in vivo study. Histol Histopathol 2009; 24: 293-8. 
16. Orhan, K, Nishiyama, H, Tadashi, S, Murakami, S, Furukawa, S. Comparison of altered signal intensity, position, and morphology of the TMJ disc in MR images corrected for variations in surface coil sensitivity. Oral Surg Oral Med Oral Pathol Oral Radiol Endod 2006; 101: 515- 22.

17. Taşkaya-Yilmaz, N, Oğütcen-Toller, M. Magnetic resonance imaging evaluation of temporomandibular joint disc deformities in relation to type of disc displacement. J Oral Maxillofac Surg 2001; 59: 860- 5.

18. Milam, SB. Pathophysiology and epidemiology of TMJ. J Musculoskelet Neuronal Interact 2003; 3: 382-90.

19. Zardeneta, G, Milam, SB, Schmitz, JP. Iron-dependent generation of free radicals: plausible mechanisms in the progressive deterioration of the temporomandibular joint. J Oral Maxillofac Surg 2000; 58: 302- 8.

20. Carlsson, GE, Oberg, T, Bergman, F, Fajers, CM. Morphological changes in the mandibular joint disk in temporomandibular joint pain dysfunction syndrome. Acta Odontol Scand 1967; 25: 163- 81.

21. Castelli, WA, Nasjleti, CE, Diaz-Perez, R, Caffesse, RG. Histopathologic findings in temporomandibular joints of aged individuals. J Prosthet Dent 1985; 53: 415- 19.

22. Helmy, ES, Timmis, DP, Sharawy, MH, Abdelatif, O, Bays, RA. Fatty change in the human temporomandibular joint disc. Light and electron microscopy study. Int J Oral Maxillofac Surg 1990; 19: 38- 43.

23. De Bont, LGM, Stengenga, B. Pathology of temporomandibular joint internal derangement and osteoarthrosis. Int J Oral Maxillofac Surg 1993; 22: 71- 4.

24. Marchetti, C, Piacentini, C, Farina, A, Bernasconi, G, Calligaro, A. A microscopic and immunocytochemical study of structural changes in dysfunctional human temporomandibular joint discs. Arch Oral Biol 1995; 40: 549- 57.

25. Milam, SB, Zardeneta, G, Schmitz, JP. Oxidative stress and degenerative temporomandibular joint disease: a proposed hypothesis. J Oral Maxillofac Surg 1998; 56: 214- 23.

26. Jibiki, M, Shimoda, S, Nakagawa, Y, Kawasaki, K, Asada, K, Ishibashi, K. Calcifications of the disc of the temporomandibular joint. J Oral Pathol Med 1999; 28: 413- 19.

27. Matsuda, S, Mishima, K, Yoshimura, Y, Hatta, T, Otani, H. Apoptosis in the development of the temporomandibular joint. Anat Embryol 1997; 196: 383- 91.

28. Nagai, H, Kumamoto, H, Fukuda, M, Takahashi, T. Inducible nitric oxide synthase and apoptosis-related factors in the synovial tissues of temporomandibular joints with internal derangement and osteoarthritis. $J$ Oral Maxillofac Surg 2003; 61: 801- 7.

29. Spears, R, Oakes, R, Bellinger, LL, Hutchins, B. Tumour necrosis factor-alpha and apoptosis in the rat temporomandibular joint. Arch Oral Biol 2003; 48: 825- 34.

30. Huang, Q, Singh, B, Sharawy, M. Immunohistochemical analysis of Bcl-2 and Bax oncoproteins in rabbit craniomandibular joint. Arch Oral Biol 2004; 49: 143-8.

31. Gu, Z, Shibata, T, Cao, Z, Feng, J, Hu, J. Chondrocyte apoptosis in temporomandibular joints with disc displacement. J Oral Maxillofac Surg 2002; 60: 1026- 31.

32. Kim, HA, Lee, YJ, Seong, SH, Choe, KW. Apoptotic chondrocyte death in human osteoarthritis. J Rheumatol 2000; 27: 455- 62.

33. Blanco, FJ, Guitian, R, Vazquez-Martul, E, de, Toro, FJ Galdo, F. Osteoarthritis chondrocytes die by apoptosis. A possible pathway for osteoarthritis pathology. Arthritis Rheum 1998; 41: 284- 9.

34. Charriaut-Marlangue, C, Ben-Ari, Y. A cautionary note on the use of the TUNEL stain to determine apoptosis. Neuroreport 1995; 7: 61-4.

35. Adams, JM, Cory, S. The Bcl-2 protein family: arbiters of cell survival. Science 1998; 281: 1322- 6.

36. Ferri, KF, Kroemer, G. Organelle-specific initiation of cell death pathways. Nat Cell Biol 2001; 3: $255-63$.

37. Green, DR, Reed, JC. Mitochondria and apoptosis. Science 1998; 281: 1309- 12.

38. Scaffidi, C, Schmitz, I, Zha, J, Korsmeyer, SJ, Krammer, PH, Peter, ME. Differential modulation of apoptosis sensitivity in CD95 type I and type II cells. J Biol Chem 1999; 274: 22532-8.

39. Krammer, PH. CD95(APO-1/Fas)-mediated apoptosis: live and let die. Adv Immunol 1999; 71: 163- 210.

40. Park, JB, Lee, JK, Park, SJ, Kim, KW, Riew, KD. Mitochondrial involvement in fas-mediated apoptosis of human lumbar disc cells. J Bone Joint Surg Am 2005; 87: 1338- 42.

41. Dimitroulis, $\mathrm{G}$. The role of surgery in the management of disorders of the temporomandibular joint: a critical review of the literature. Part 2. Int J Oral Maxillofac Surg 2005; 34: 231- 7. 
42. Dolwick, MF. Temporomandibular joint surgery for internal derangement. Dent Clin North Am 2007; 51 : 195208.

43. Miloro, $M$, Henriksen, $B$ Discectomy as the primary surgical option for internal derangement of the temporomandibular joint. J Oral Maxillofac Surg 2010; 68: 782-9.

44. Touré, G, Duboucher, $C$, Vacher, C. Anatomical modifications of the temporomandibular joint during ageing. Surg Radiol Anat 2005; 27: 51- 5.

45. Kopp, S. Clinical findings in temporomandibular joint osteoarthrosis. Scand J Dent Res 1977; 85: 434- 43.

46. Luder, HU. Factors affecting degeneration in human temporomandibular joints as assessed histologically. Eur J Oral Sci 2002; 110: 106- 13.

47. Shafer, DM, Assael, L, White, LB, Rossomando, EF. Tumor necrosis factor- $\alpha$ as a biochemical marker of pain and outcome in temporomandibular joint with internal derangement. J Oral Maxillofac Surg 1994; 52: 78691.

48. Kubota, E, Kubota, T, Matsumoto, J, Shibata, T, Muratami, KI. Synovial fluid cytokines and proteinases as markers of temporomandibular joint disease. J Oral Maxillofac Surg 1998; 56: 192- 8.

49. Kaneyama, K, Segami, N, Nishimura, M, Suzuki, T, Sato, J. Importance of proinflammatory cytokines in synovial fluid from 121 joints with temporomandibular disorders. Br J Oral Maxillofac Surg 2002; 40: 41823.

50. Nishimura, M, Segami, N, Kaneyama, K, Sato, J, Fujimura, K. Comparison of cytokine level in synovial fluid between successful and unsuccessful cases in arthrocentesis of the temporomandibular joint. $J$ Oral Maxillofac 2004; 62: 284- 7.

51. Kardel, R, Ulfgren, AK, Reinholt, P, Holmlund, A. Inflammatory cell and cytokine patterns in patients with painful clicking and osteoarthritis in temporomandibular joint. Int J Oral Maxillofac Surg 2003; 32: 390- 6.

52. Suzuki, T, Segami, N, Nishimura, M, Nojima, T. Co-expression of interleukin-1 $\beta$ and tumor necrosis factor $\alpha$ in synovial tissues and synovial fluids of temporomandibular joint with internal derangement: comparison with histological grading of synovial inflammation. J Oral Pathol Med 2002; 31: 549- 57.

53. Creagh, EM, Conroy, H, Martin, SJ. Caspase-activation pathways in apoptosis and immunity. Immunol Rev 2003; 193: 10- 21.

54. Moncada, S, Palmer, RM, Higgs, EA. Nitric oxide: physiology, pathophysiology, and pharmacology. Pharmacol Rev 1991; 43: 109- 42.

55. Schmidt, HH, Walter, U. NO at work. Cell 1994; 78: 919- 25.

56. Brockhaus, F, Brüne, B. p53 accumulation in apoptotic macrophages is an energy demanding process that precedes cytochrome $c$ release in response to nitric oxide. Oncogene 1999; 18: 6403- 10.

57. Brüne, B, Von Knethen, A, Sandau, KB. Nitric oxide (NO): an effector of apoptosis. Cell Death Differ 1999; 6 : 969- 75.

58. Blanco, FJ, Ochs, RI, Schwarz, H, Lotz, M. Chondrocyte apoptosis induced by nitric oxide. Am J Pathol 1995; 146: $75-85$.

59. Hashimoto, S, Takahashi, K, Amiel, D, Coutts, RD, Lotz, M. Chondrocyte apoptosis and nitric oxide production during experimentally induced osteoarthritis. Arthritis Rheum 1998; 41: 1266- 74.

60. Erickson, GF. Defining apoptosis: players and systems. J Soc Gynecol Invest 1997; 4: 219- 28.

61. Huppertz, B, Kaufmann, HGFP. The apoptosis cascade-morphological and immunohistochemical methods for its visualization. Anat Embryol 1999; 200: 1- 18.

62. Sukedai, M, Fukuda, J. Involvement of tumor necrosis factor-alpha and interleukin-8 in antigen induced arthritis of the rabbit temporomandibular joint. J Oral Pathol Med 2004; 33: 102- 10.

63. Imirzalioğlu, P, Uçkan, S, Güler, N, Haberal, A, Uçkan, D. Synovial apoptosis in temporomandibular joint disc displacement without reduction. Oral Surg Oral Med Oral Pathol Oral Radiol Endod 2009; 108: 693- 8.

64. Tschoeke, SK, Hellmuth, M, Hostmann, A, et al. Apoptosis of human intervertebral discs after trauma compares to degenerated discs involving both receptor-mediated and mitochondrial-dependent pathways. J Orthop Res 2008; 26: 999- 1006.

65. Gruber, HE, Hanley, EN. Analysis of aging and degeneration of the human intervertebral disc. Comparison of surgical specimens with normal controls. Spine 1998; 23: 751- 7. 
66. Park, JB, Chang, H, Kim, KW. Expression of Fas ligand and apoptosis of disc cells in herniated lumbar disc tissue. Spine 2001; 6: 618- 21.

67. Ward, C, Dransfield, I, Chilvers, ER, Haslett, C, Rossi, AG. Pharmacological manipulation of granulocyte apoptosis: potential therapeutic targets. Trends Pharmacol Sci 1999; 20: 503-9.

68. Rannou, F, Lee, TS, Zhou, RH, et al. Intervertebral disc degeneration: the role of the mitochondrial pathway in annulus fibrosus cell apoptosis induced by overload. Am J Pathol 2004; 164: 915- 24.

69. Haschtmann, D, Stoyanov, JV, Gédet, P, Ferguson, SJ. Vertebral endplate trauma induces disc cell apoptosis and promotes organ degeneration in vitro. Eur Spine J 2008; 17: 289- 99.

70. Leonardi, R, Almeida, LE, Trevilatto, PC, Loreto, C. Occurence and regional distribution of TRAIL and DR5 on temporomandibular joint discs: comparison of disc derangement with and without reduction. Oral Surg Oral Med Oral Pathol Oral Radiol Endod 2010; 109: 244- 51.

71. Ariga, K, Miyamoto, S, Nakase, T, et al. The relationship between apoptosis of endplate chondrocytes and aging and degeneration of the intervertebral disc. Spine 2001; 15: 2414- 20.

72. Lotz, JC, Chin, JR. Intervertebral disc cell death is dependent on the magnitudeand duration of spinal loading. Spine 2000; 25: 1477-83. 